\title{
A Smart Home Center Platform Solution Based on Smart Mirror
}

\author{
Xibo DENG ${ }^{1}$, Zhiran $\mathrm{PENG}^{2}$, and Wenquan $\mathrm{WU}^{1}$ \\ ${ }^{1}$ School of Electronic Engineering, Navy University of Engineering, Wuhan, 430000, P.R. China \\ ${ }^{2}$ School of Electrical Engineering, Navy University of Engineering, Wuhan, 430000, P.R. China
}

\begin{abstract}
With the popularization of the concept of smart home, people have raised requirements on the experience of smart living. A smart home platform center solution is put forward in order to solve the intelligent interoperability and information integration of smart home, which enable people to have a more intelligent and convenient life experience. This platform center is achieved through the Smart Mirror. The Smart Mirror refers to a smart furniture, on the basis of the traditional concept of mirror, combining Raspberry Pi, the application of one-way mirror imaging principle, the touch - enabled design, voice and video interaction. Smart Mirror can provide a series of intelligent experience for the residents, such as controlling all the intelligent furniture through Smart Mirror; accessing and displaying the weather, time, news and other life information; monitoring the home environment; remote interconnection operation.
\end{abstract}

\section{The overall framework and functionality of the solution}

Smart home is intelligent home. In the current smart home solutions, the vast majority of them are through the embedded gateway or independent central processor to communicate with the outside, relying on the Internet of things(IoT), all kinds of household things linked together. Smart home does not have a complete system of technical standards and solutions in the international and domestic [1]. But with the development of Smart home, more and more users need an integrated system platform, which can provide a convenient control operation and human-computer interaction for people. At the same time, it also provides people with various social life information. This article is based on the mirror used in home life, designed and implemented a Smart Mirror, which based on the home center intelligent platform solutions. This center platform includes showing realtime weather, news, monitoring of family health, monitoring of home environments, burglar alarm and so on. Not only that, all the home appliances and related intelligent devices access to the Internet, real-time feedback home status parameters.

Nowadays, all household appliances have corresponding intelligent products in connection with LAN or Internet, such as small alarm clock, dust collector, television, washing machine, with different grades at different prices. The mirror is used as the solution to satisfy people's demands for intelligent home. Main reasons are shown as follows:
(1) As the mirror usually hangs in contrast with other electrical appliances, it can spread throughout each independent living space.

(2) As the natural imaging curtain, the mirror occupies a small space, which is convenient for imaging and saves the living space at home.

(3) Different from other large household appliances, the mirror is not limited by space, and can meet different requirements in different environments. It is advantageous in integrating different functions in different laying space. Three typical applications are taken as an example to elaborate overall framework of function and solution as Fig. 1.

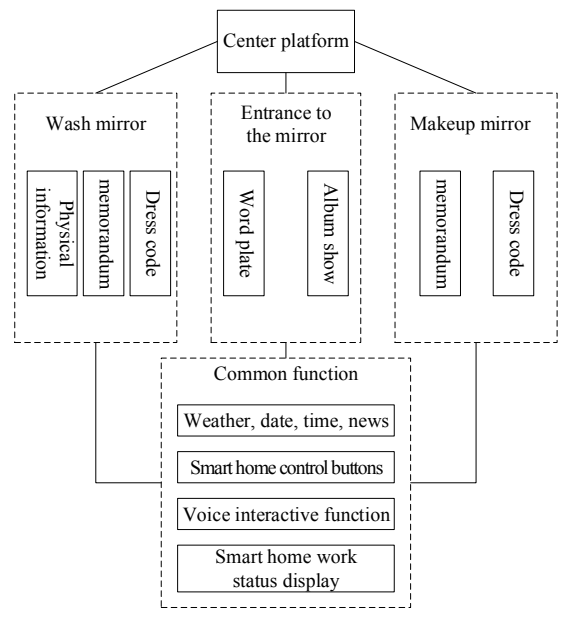

Figure 1. Intelligent mirror center platform overall framework

\footnotetext{
* Corresponding author: 347718046@qq.com
} 


\section{Hardware design}

\subsection{Raspberry $\mathrm{Pi}$}

Raspberry Pi was developed by British charitable organization Raspberry Pi foundation. Eben Upton of University of Cambridge officially launched computer version of Raspberry Pi on the basis of Linux in March 2012 [2]. Raspberry Pi was originally designed for the students in computer programming education, but admired by numerous computer fanciers after coming out. It is a micro computer motherboard based on ARM, with SD/ Micro SD as storage hard disk, in connection with keyboard, mouse and cable, furnished with TV output interface with video analog signal and HDMI HD video output interface. These components are integrated in the motherboard slightly larger than credit card, with all the basic functions of $\mathrm{PC}$ [3]. There are $\mathrm{A} / \mathrm{A}+/ \mathrm{B} / \mathrm{B}+/$ second $\mathrm{B}$ type /third $\mathrm{B}$ type, and three kinds of internal storage, including $256 \mathrm{MB}, 512 \mathrm{MB}$ and $1 \mathrm{~GB}$. Raspberry Pi takes Python as the main programming language. The paper is based on "Raspberry $\mathrm{Pi} \mathrm{B}+$ " released in July 2014, which was equipped with internal storage of $512 \mathrm{MB}, 40$ pins of GPIO and 4 USB interfaces. In contrast with the previous generation, the new one renovates hot plug and over-current protection, replaces old SD socket with Micro SD, reduces power consumption to $0.5 \sim 1 \mathrm{~W}$, and adopts low-noise power supply in audio circuit [4]. Contrasting with taking micro-controller [5], no much extra money is spent on developing Raspberry $\mathrm{Pi}$, and peripheral circuit design is saved, which means that it is a good choice to create excellent multimedia center.

\subsection{Imaging control system}

The imaging control system lays foundation for display of Smart Mirror and human-computer interaction. In addition to retention of imaging characteristics of the mirror, daily information, such as weather, news and time, is obtained directly by the Internet. At the same time, synchronously memo, changing of teleprompter board and monitoring the mirror image can be achieved through the user's mobile phone. Users can even directly control the intelligent home, selfie in front of the mirror and look through built-in family photos through directly touching. The imaging control system is mainly based on display technology, by which expected information and control unit are obtained in home mirror. The imaging control system of Smart Mirror is shown by Fig. 2, mainly consisting of following parts: unidirectional mirror, driving plate, display screen and infrared frame.

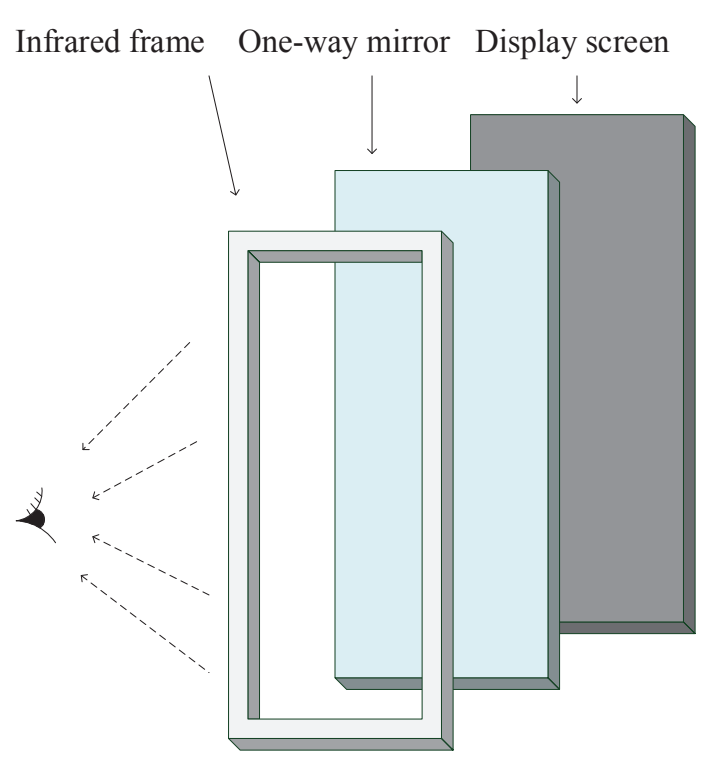

Figure 2. Imaging system structure

\subsubsection{One-way mirror}

A one-way mirror is also called single lens, atomic mirror. According to the principle of human eye capturing high light objects in visual range, black room environment is constructed on one side of mirror, supplemented by high light on the other side. In this case, people on high light side take glass as a mirror, but look objects though glass in the dark environment. The specific observation environment is demonstrated in Fig. 3. Currently, the one-way mirror adopts magnetron sputtering coating technology, with thin semi reflective layer in the middle of two pieces of glass attached by PVB glue, as shown in Fig. 4.

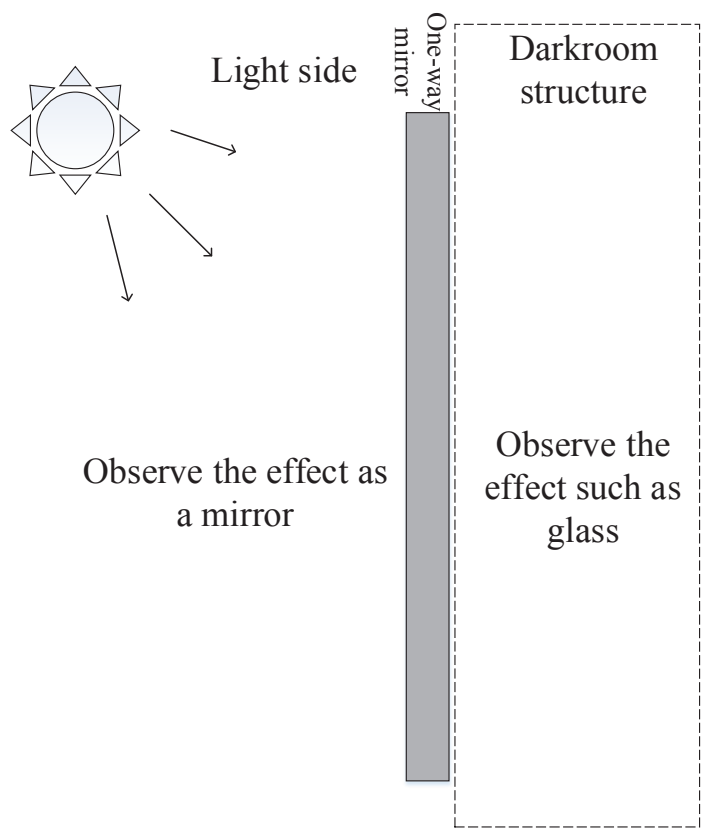

Figure 3. Imaging system structure 


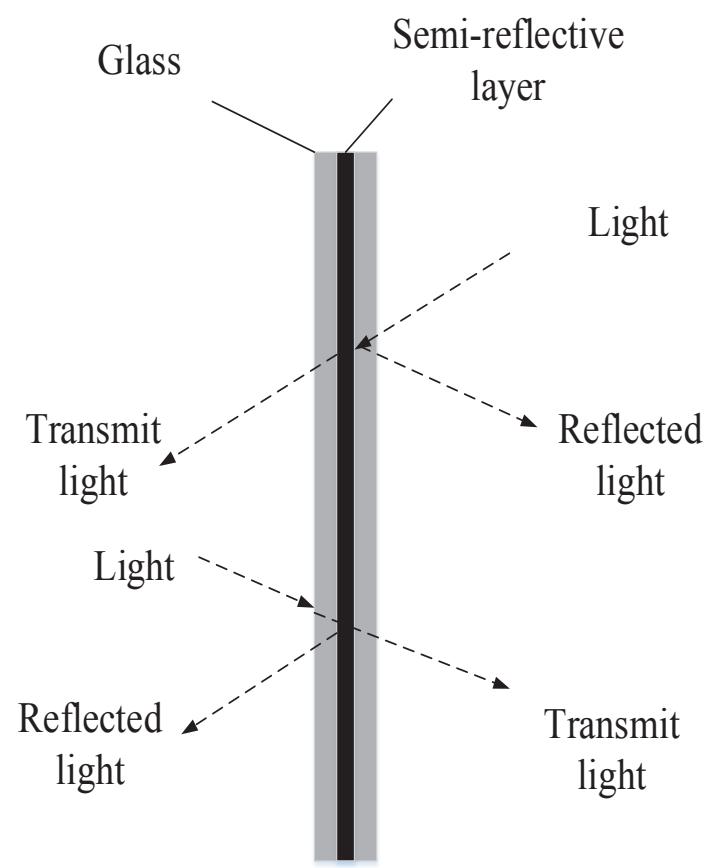

Figure 4. One-way mirror structure

\subsubsection{Display screen}

In the term of selection of screen, this paper chooses TFT type liquid crystal display after taking both contrast and color saturation into account [6]. This kind of monitor includes liquid crystal materials, thin film transistor, polarizing plate, light guide plate, which can display graphics in full color, and ensure $70 \%$ of all black display at high utilization rate through interface design. This design is conducive to the chamber structure behind the mirror, realizing mirror imaging effect [7].

\subsubsection{General drive board}

The generic driver board is also known as the LCD driver board. There are a lot of brands of common driver plate in market, such as Leroy, DingKe, Triumph and Yuekang. Although components of general driver board are inconsistent with those of original driver board, as long as by drivers are loaded into general drive board by LCD programmer and lines are modified, different LCD screens can be driven, which reduces costs very low cost, is easy to develop and accepted by the customers.

\subsubsection{Infrared frame}

In order to solve the basic problem of touch interaction, infrared frame is adopted, which can connect with Raspberry Pi through the USB port. Infrared network is constructed inside the infrared range, and determines the touch position through touching objects, so as to achieve the touch interaction purpose.

\section{Implementation plan}

\subsection{Interactive function implementation}

\subsubsection{Touch interaction}

The touch control mode of Smart Mirror is realized by the infrared frame. The interaction solution is simple, which only needs to carry on the related position debugging after being connected with the infrared frame. Besides, the design is less demanded on the mirror itself, the mirror can be developed on the basis of the existing without using those expensive capacitive touch screens or resistance touch screens. In the meanwhile, the infrared frame mode requires less on the user's touch side compared with the capacitive touch screens or resistance touch screens, wet fingers, non-conductor touch bar, and any other thin rod shape objects are available with the Smart Mirror.

\subsubsection{Voice interaction}

At present, the development of cloud computing enables many terminal devices to interact with each other by cloud computing efficiently and accurately [8]. In the voice interaction part, this solution is based on the open source development platform of Baidu Voice Assistant, setting the voice recognition part in the cloud, and the voice recognition part is accomplished off line. The development interface is shown in Fig. 5. The benefits are mainly embodied in three following aspects: 1 . The response mode is fixed, family members can set on their own mobile phones or PCs; 2. Processing speed is faster than traditional speech recognition, comparison test results are shown in Fig. 6; 3. Making the central platform safer, closed-source voice response processing makes the Smart Mirror emerging in the network security protection.

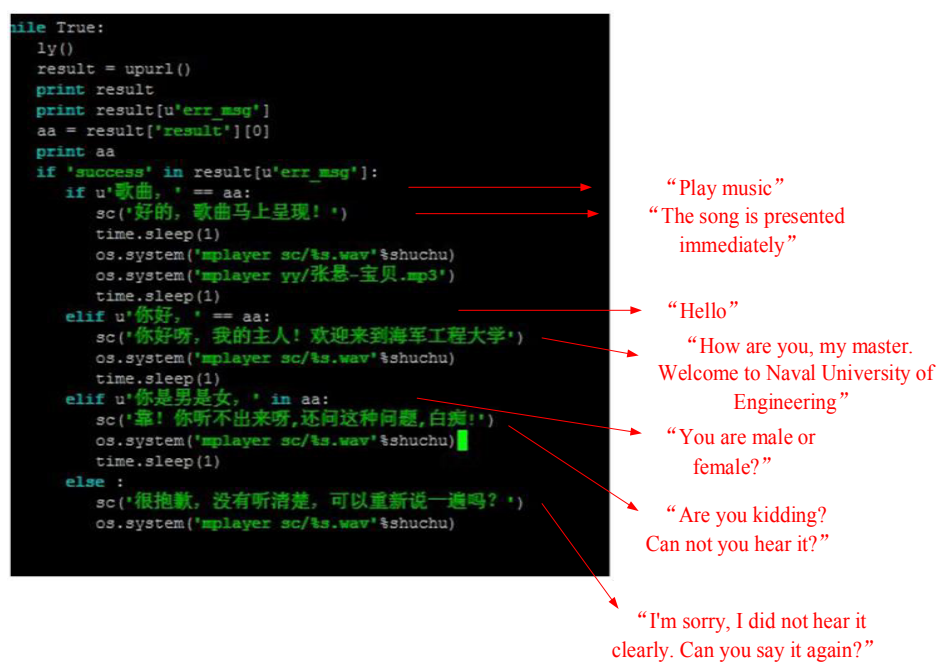

Figure 5. Voice processing 


\section{Response time comparison}

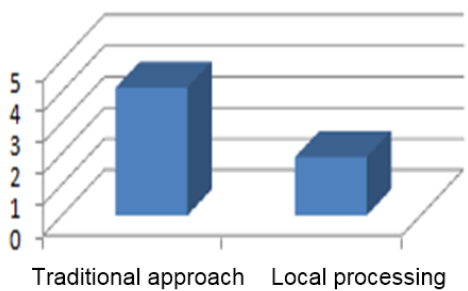

n Time $(s)$

Figure 6. Response time comparison

\subsection{Infrared wake up}

The infrared wake-up function is effective in two aspects, user experience and energy saving. Raspberry Pi with a dedicated external infrared sensor module can make the infrared sensor through the Raspberry Pi by pin. The infrared control terminal is opened by the Raspberry Pi program, allowing the Smart Mirror wake up when people move or moving subjects are in front of the mirror, when the mirror is unmanned, the mirror acts as a common one.

\subsection{Anti - theft monitoring and image acquisition}

The Raspberry Pi used as the central processing unit has 4 USB communication ports, one of which is assigned to the camera so that the mirror has the function of capturing the scene in front of the mirror in real time. Based on the open source public number development platform of WeChat public client, the home environment can be real-time monitored by mobile digital terminals such as mobile phone, and users can decide the face recognition mode positive or not to control the opening of the intelligent function, which can increase the safety of home furnishing equipment.

\subsection{The design of portable terminal}

The solution proposed in this paper is not only valid in the development and function integration of intelligent home furnishing, but also provides a more convenient and fresher way for user's control. The main method is based on the development of mobile digital terminals and the intelligent home furnishing network. Nowadays, Nowadays, the electronic device that most people cannot do without in daily life is the mobile phone, laptop, tablet computer and vehicle receiving terminals are also included, with the help of them, people can synchronize daily reminders, refresh WeChat public service number information, control others intelligent home furnishings and so on. The interfaces are shown as Fig.7 and Fig.8.

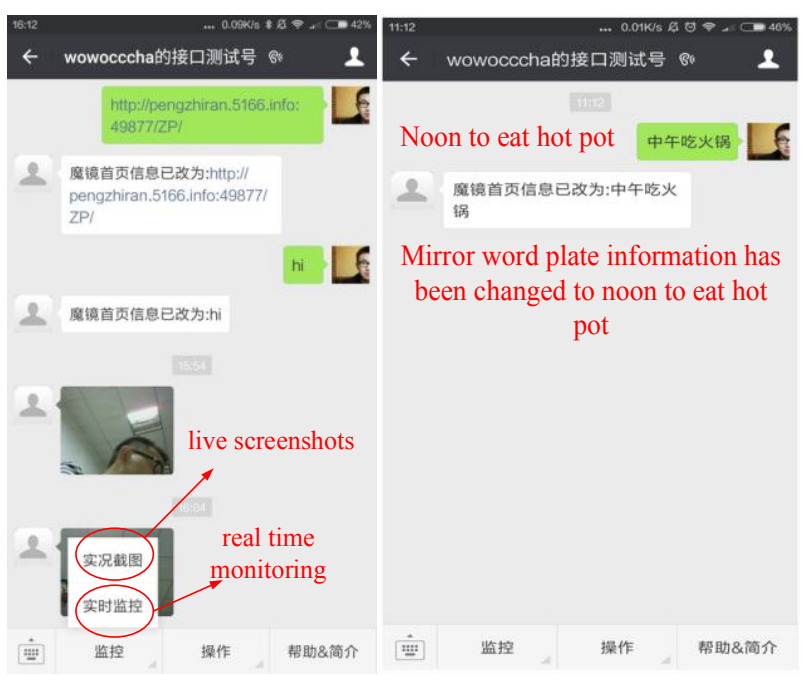

Figure 7. WeChat platform development

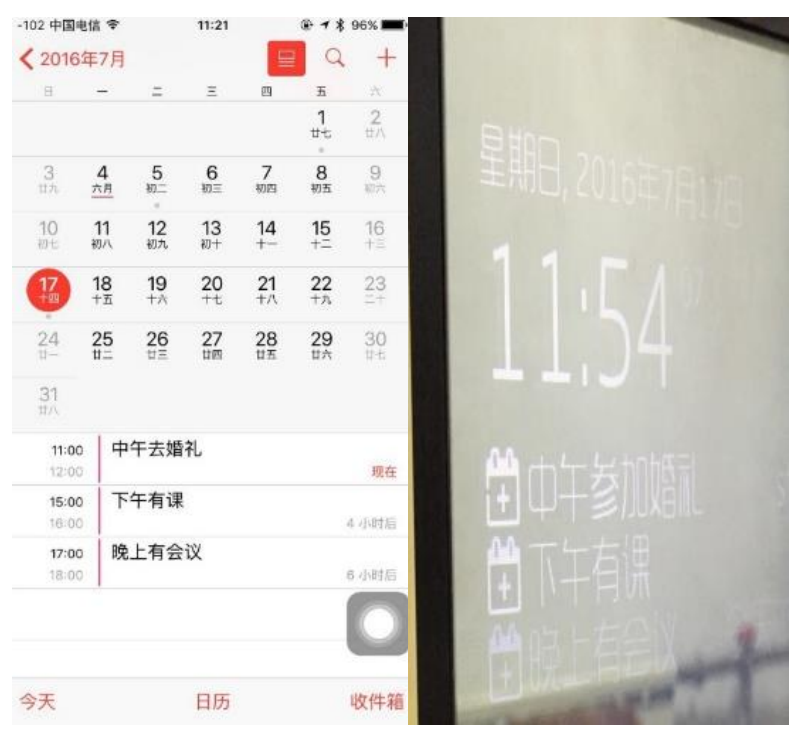

Figure 8. Memo sync

\section{Software design}

To carry out the relevant preparatory work in the development platform before the software design. The specific tasks are as follows: 1.The solution uses a $2 \mathrm{G}$ SD card, preferably a high-speed card that recommended more than Class4, due to the card directly affects the speed of Raspberry Pi faction running. 2. Download the Raspberry Pi system imaging file. 3. Configure the SD card, connect the keyboard and keyboard, power cord, HDMI cable and other hardware devices.

As a result of daily household life, intelligent home system of the various parts of the work is relatively independent [9], so in this solution the application is modular design. According to the smart home center platform of the various requirements, the system workflow of Smart Mirror is shown in Fig. 9. 


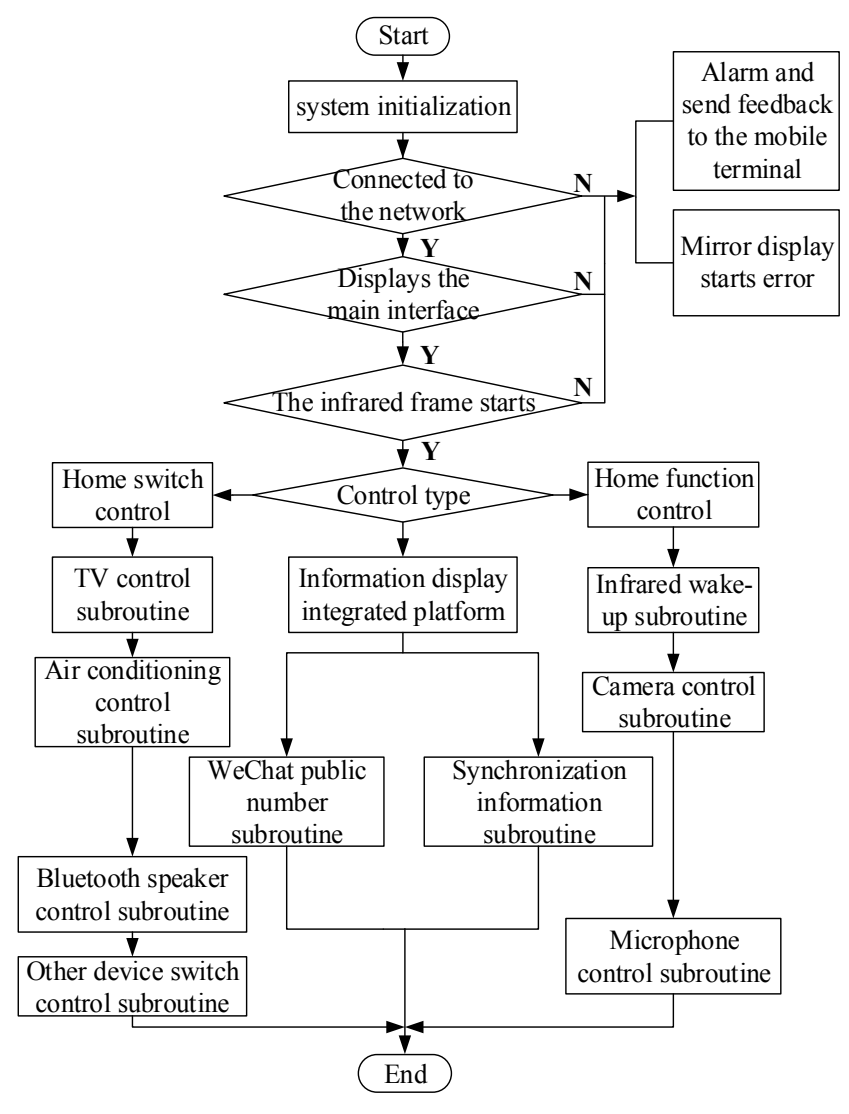

Figure. 9. System software design process

\section{5 summary}

Smart Mirror, as a solution of smart home center platform, can provide a physical terminal to the user in the operation and experience. At the same time without additional furniture, under the premise of efficient and intelligent it can provide users with high quality of life. Smart Mirror as a home center platform has many incomparable advantages which other devices do not have, such as relatively low cost, do not need additional development of the display screen. In addition, it does not contain the electromechanical system, so the external control system development is simple and so on. Overall, the Smart Mirror can provide a central platform for smart home in the development, application and so on. Based on this concept and design principles we designed the" Smart Magic Mirror" works and won the" first prize" in the national final teams of the eleventh "Huawei Cup" The China Graduate Electronics Design Contest; personal "second prize"; "first prize" in the national finals Smart Home Group of 2017 National College Students Connected Smarter System Innovation Competition. And the project is currently pending patent applications.

\section{References}

1. Zhang Weihua, Huang Xiaolin, Research on Intelligent Home Technology and Standardization of
Internet of Things [J]. Information Technology \& Standardization, 2012, 7: 35-38.

2. Liu Jiyuan, Application of Internet of Things Based on Raspberry [J]. Electronic World, 2016 (8): 24-25.

3. DAI Yupei, ZHANG Yu, LIU Yunfei, Intelligent home and building real-time monitoring system design $[\mathrm{J}]$. MCU and embedded systems applications, 2017, 17 (2): 49-52.

4. Hao Linwei, Liang Ying, Research and Practical Application of Network Monitoring and Home Control System Based on Raspberry + Cloud Server [J]. Internet of Things Technology, 2016, 6 (9): 4547

5. Zeng Guijing, Song Yue, He Zhihui, Hardware Design of a Intelligent Home Remote Control System [J]. Electronic Technology Applications, 2011, 37 (4): 81-84.

6. Huang ximin, TFT LCD - Future mainstream display technology $[\mathrm{J}]$. World Electronic Components, 2001 (11): 9-10.

7. Li Lianyi, TFT-LCD liquid crystal display technology and application [J]. Journal of Shaoguan University, 2012, 33 (4): 46-49.

8. Chen Wei, Qin Huibin, Cao Shuguang, etc. Based on the Android platform intelligent home system design $[\mathrm{J}]$. Electronic Technology Applications, 2015, 41 (10): 158-160.

9. Xin Hai-liang, Zhong Peisi, Zhu Shaoyi, et al. Intelligent home control system based on ZigBee [J] Electronic Technology Applications, 2013, 39 (12): $79-81$ 